Annuaire suisse de politique de développement

25-1 | 2006

Faits et statistiques 2006

\title{
6. Commerce mondial
}

Nadine Keim

\section{OpenEdition}

\section{Journals}

Édition électronique

URL : http://journals.openedition.org/aspd/317

DOI : $10.4000 /$ aspd.317

ISSN : 1663-9669

\section{Éditeur}

Institut de hautes études internationales et du développement

\section{Édition imprimée}

Date de publication : 1 avril 2006

Pagination : 79-98

ISBN : 2-88247-061-4

ISSN : 1660-5934

\section{Référence électronique}

Nadine Keim, «6. Commerce mondial », Annuaire suisse de politique de développement [En ligne], 25-1 | 2006, mis en ligne le 10 février 2010, consulté le 08 septembre 2020. URL : http:// journals.openedition.org/aspd/317; DOI : https://doi.org/10.4000/aspd.317 


\section{Commerce mondial*}

$L$ E COMMERCE des marchandises a connu une croissance spectaculaire durant l'année 2004, de $9 \%$ en volume et de $21 \%$ en valeur, soit le plus fort accroissement depuis 1979. Si l'Amérique du Nord et l'Union européenne ont consolidé leur position dominante, assurant près des deux tiers du commerce mondial des produits manufacturés, la Suisse perd progressivement sa place parmi les acteurs les plus importants du commerce mondial. Un autre élément marquant de l'année a été la croissance économique exceptionnelle de la Chine et de l'Inde et leur montée en force dans le commerce mondial.

Concernant les secteurs du textile et de l'habillement, 2005 a constitué une nouvelle étape dans leur libéralisation, avec la fin de l'accord multifibres et la levée des quotas d'importation. Il en résulte une guerre commerciale entre, d'un côté, la Chine, l'Inde et Pakistan et, de l'autre, des pays pauvres comme le Bangladesh et le Cambodge, ainsi que les Etats-Unis et l'Union européenne.

Le nouveau directeur général de l'OMC, Pascal Lamy, qui a pris ses fonctions le $1^{e r}$ septembre 2005, s'est efforcé de redynamiser les négociations commerciales, bloquées depuis l'accord de juillet 2004. Les ambitions de la Conférence ministérielle de Hongkong, qui s'est tenue du 13 au 18 décembre, ont dû être revues à la baisse. Les seuls résultats concrets ont été l'adoption d'un "paquet du développement» pour les pays les moins avancés et la fixation d'une date limite pour la suppression des subventions à l'exportation. Les négociations ont notamment buté sur la compréhension de ce qu'est ou devrait être un "cycle du développement». Pour les uns, dont la Suisse, cela passe avant tout par une libéralisation des marchés; pour d'autres, par l'octroi d'un vrai «espace politique».

En 2005, l'OMC a fêté son dixième anniversaire. Les différentes manifestations organisées à cette occasion ont permis de montrer que les blocages dont elle souffre sont également le reflet d'un certain nombre de mutations plus fondamentales: nouvelle donne géopolitique avec la montée en puissance des pays en développement, complexification des jeux d'alliance au-delà des clivages NordSud, grippages institutionnels. Quant à la société civile, elle a été très active en 2005 dans le domaine du commerce international.

\subsection{Evolution des relations commerciales}

L'année 2004 a été marquée par une croissance spectaculaire du commerce, dans un contexte de forte croissance de la production ${ }^{1}$. Le volume des exportations de marchandises a progressé de 9\%, le meilleur résultat depuis 2000. Cette accélération est largement imputable aux très bons résultats du commerce des produits manufacturés $(+10 \%)$, soit un rythme deux fois plus rapide qu'en 2003. La croissance des échanges a été supérieure à celle de la production.

* Par Nadine Keim, lic. ès sc. pol., consultante indépendante.

1 OMC, Statistiques du commerce international 2005, Genève, 2005. Voir aussi OMC, Rapport sur le commerce mondial 2005, Genève, 2005. 
Tableau 13: Exportations mondiales de marchandises et de services commerciaux, 2002-2004 (en milliards de dollars)

\begin{tabular}{llll}
\hline & $\mathbf{2 0 0 4}$ & $\mathbf{2 0 0 2}$ & $\mathbf{2 0 0 3}$ \\
\hline Marchandises & 8907 & 6270 & 7274 \\
\hline Services commerciaux & 2125 & 1570 & 1763 \\
\hline
\end{tabular}

Source: OMC, Statistiques du commerce international 2005, Genève, 2005, tableau 1, p. 3.

En 2004, la valeur en dollars du commerce mondial des marchandises a fait un bond de $21 \%$, atteignant 8907 milliards de dollars, ce qui représente le plus fort accroissement annuel en valeur depuis 1979. Cette évolution est due principalement à une forte augmentation des prix - conjuguée à une nouvelle dépréciation (9\%) du dollar américain face aux monnaies des grandes nations commerçantes d'Europe -, en particulier celle des prix du pétrole qui n'ont pas atteint un niveau aussi élevé depuis vingt ans. La valeur des exportations de combustibles et autres produits des industries extractives s'est accrue de $30 \%$ à $45 \%$ en 2004 et, comme l'année précédente, leur expansion a été nettement plus rapide que celle du commerce des produits manufacturés $(+20 \%)$ ou agricoles $(+15 \%)$. Les échanges de services commerciaux se sont accrus en termes nominaux de $18 \%$ pour s'établir à 2125 milliards de dollars en 2004.

On s'attend à ce que le recul de la production économique, dû en partie à la forte hausse des prix du pétrole, ralentisse la croissance du commerce mondial en 2005. Les exportations mondiales de marchandises devraient augmenter de $6,5 \%$ en 2005, soit nettement moins que la croissance spectaculaire de l'année précédente ${ }^{2}$.

Tableau 14: Croissance de la valeur du commerce mondial des marchandises, par régions, 2004 (en milliards de dollars et en pourcentage)

\begin{tabular}{|c|c|c|c|c|c|c|c|c|}
\hline \multirow{3}{*}{ Région } & \multicolumn{4}{|c|}{ Exportations } & \multicolumn{4}{|c|}{ Importations } \\
\hline & \multirow{2}{*}{$\begin{array}{l}\text { Valeur } \\
2004\end{array}$} & \multicolumn{3}{|c|}{ Variation annuelle (\%) } & \multirow{2}{*}{$\begin{array}{c}\text { Valeur } \\
2004\end{array}$} & \multicolumn{3}{|c|}{ Variation annuelle (\%) } \\
\hline & & $2000-04$ & 2003 & 2004 & & 2000-04 & 2003 & 2004 \\
\hline Monde & 8907 & 9 & 17 & 21 & 9250 & 9 & 17 & 21 \\
\hline Amérique du Nord & 1324 & 2 & 5 & 14 & 2013 & 5 & 8 & 17 \\
\hline Amérique latine et centrale & 276 & 9 & 13 & 30 & 237 & 4 & 5 & 27 \\
\hline Europe & 4031 & 11 & 19 & 19 & 4140 & 11 & 20 & 20 \\
\hline Union européenne (25) & 3714 & 11 & 19 & 19 & 3791 & 10 & 20 & 19 \\
\hline $\begin{array}{l}\text { Communauté des Etats } \\
\text { indépendants }\end{array}$ & 266 & 16 & 27 & 37 & 172 & 21 & 27 & 30 \\
\hline Russie & 183 & 15 & 27 & 35 & 96 & 21 & 25 & 27 \\
\hline Afrique & 232 & 12 & 25 & 32 & 212 & 13 & 22 & 27 \\
\hline Moyen-Orient & 390 & 10 & 20 & 29 & 252 & 12 & 13 & 27 \\
\hline Asie & 2388 & 10 & 18 & 25 & 2224 & 10 & 19 & 27 \\
\hline Chine & 593 & 24 & 35 & 35 & 561 & 26 & 40 & 36 \\
\hline Japon & 566 & 4 & 13 & 20 & 455 & 5 & 14 & 19 \\
\hline $\begin{array}{l}\text { Six pays commerçants } \\
\text { d'Asie de l'Est }\end{array}$ & 860 & 7 & 15 & 24 & 785 & 6 & 12 & 27 \\
\hline
\end{tabular}

Source: OMC, Statistiques du commerce international 2005, Genève, 2005, tableau I.3, p. 22.

Remarque: La différence entre la valeur des exportations mondiales et celle des importations mondiales se justifie par la prise en compte des frais de port et d'assurance dans le total des importations (cost of insurance and freight, CIF).

2 OMC, Statistiques du commerce. La croissance du commerce devrait ralentir en 2005 par rapport au rythme record de 2004, communiqué de presse, n 417, 27 octobre 2005. 
Dans le classement 2004 des principaux pays exportateurs et importateurs, la Suisse a disparu de la liste des 20 premiers pays exportateurs au monde $\left(21^{\mathrm{e}}\right.$ place en 2004, alors qu'elle se situait encore à la $19^{\mathrm{e}}$ place en 2003). Par contre, elle maintient en 2004, comme en 2003, sa $18^{\mathrm{e}}$ position en ce qui concerne les importations. En termes de services, la Suisse se situe au $18^{\mathrm{e}}$ rang des principaux pays exportateurs (16 $\mathrm{e}$ place en 2003) et au $27^{\mathrm{e}}$ rang en ce qui concerne les importations. La Suisse perd ainsi progressivement sa place parmi les acteurs les importants du commerce mondial.

\subsubsection{Commerce mondial des marchandises par régions}

La plupart des grandes régions ont enregistré en 2004 une forte croissance économique, qui a permis une expansion vigoureuse du commerce mondial. Cette expansion est surtout due à la reprise des exportations de produits manufacturés de l'Amérique du Nord (9\%) et de l'Union européenne (7\%). Ensemble, ces deux régions assurent près des deux tiers du commerce mondial des produits manufacturés.

Un fait marquant en 2004 a été que les deux pays les plus peuplés de la planète, la Chine et l'Inde, ont enregistré une croissance économique exceptionnelle (9,5\% et $7,3 \%$ respectivement) et ont vu leurs échanges progresser très fortement pour la deuxième année consécutive. Les régions exportatrices de pétrole (la Communauté des Etats indépendants, le Moyen-Orient et l'Afrique) ont vu leurs exportations de marchandises s'accroître à un rythme beaucoup plus rapide que la moyenne mondiale.

Les exportations de marchandises des pays les moins avancés (PMA) ont augmenté d'un tiers, pour passer à 62 milliards de dollars en 2004. La hausse des prix des produits de base et l'augmentation du volume de pétrole brut ont contribué à ce bon résultat. Les PMA non exportateurs de pétrole ont enregistré une croissance des exportations inférieure à la moyenne du groupe.

Mis à part la hausse du prix des produits pétroliers, l'autre événement significatif en 2004 a été la confirmation de l'émergence de la Chine en tant qu'importateur et fournisseur de biens et services. La croissance dynamique et soutenue de ses exportations et importations au cours de ces dernières années a placé la Chine au troisième rang des grandes puissances commerciales en 2004. La Chine est même devenue le premier importateur mondial de nombreux produits de base et le premier fournisseur mondial d'un certain nombre de produits manufacturés. La progression rapide de la Chine dans le commerce mondial crée des opportunités pour de nombreux pays, mais impose également des ajustements à d'autres, notamment dans le domaine des textiles. 
Le $1^{\text {er }}$ janvier 2005 a marqué une nouvelle étape dans la libéralisation du commerce mondial avec la levée des quotas d'importation dans les secteurs du textile et de l'habillement (dénommés «quotas» ci-dessous) par les Etats-Unis, l'Union européenne et le Canada. Les grands acheteurs de vêtements ne sont, en principe, plus contraints par les quotas de disperser leurs commandes aux quatre coins du monde, y compris dans les pays les moins avancés. Cette étape tant attendue aurait dû signifier la victoire de la libéralisation des échanges sur les velléités protectionnistes et l'opacité du système des quotas. En réalité, le jeu reste faussé; plusieurs pays pauvres, pour qui le textile est la seule porte d'entrée vers l'industrialisation, vont être éliminés au profit de quelques grands pays en plein développement (voir encadré ci-dessous).

\section{Quotas et Accord sur les textiles}

Le premier Arrangement multifibres (AMF), entré en vigueur en 1974, introduisait un système de quotas pour protéger les grands marchés occidentaux des exportations de textile et d'habillement en provenance des pays en développement. Prolongé à trois reprises, I'AMF a provoqué l'éclatement actuel de la chaîne de production mondiale, car il a contraint les multinationales du textile à éparpiller leurs sources d'approvisionnement et de fabrication, alors que leurs intérêts étaient de concentrer la majorité de leurs sous-traitants dans les pays affichant les avantages compétitifs les plus attrayants.

En 1995, I'AMF est remplacé par l'Accord sur les textiles et les vêtements (ATV), accord qui fut négocié dans le cadre de I'Uruguay Round du GATT (1986 à 1994). Cet accord visait le démantèlement des quotas en dix ans et établissait un calendrier de quatre étapes successives, censé rendre le processus de libéralisation moins douloureux. Toutefois, les pays importateurs ne s'en serviront que pour gagner du temps, en repoussant à la fin de l'échéance la levée des quotas sur les produits les plus sensibles.

En outre, I'ATV prévoit une clause de sauvegarde transitoire qui permet à un pays de se protéger contre une augmentation des importations d'un produit telle qu'elle porte ou menace de porter un «préjudice grave " à l'industrie domestique concernée. Acceptée par tous les pays membres de l'Organisation mondiale du commerce (OMC), cette clause peut être appliquée sur une base sélective, pays par pays, par accord mutuel, ou de façon unilatérale dans le cas où aucun accord n'intervient au bout de soixante jours. Toutefois, cette clause ne peut s'appliquer pendant plus de trois ans, c'est à dire jusqu'en 2008 au plus tard.

II faut noter que les droits de douane constituent un autre système de protection. De $12 \%$ dans I'Union européenne, $15 \%$ aux Etats-Unis ou $29 \%$ en moyenne dans les pays en développement, les tarifs douaniers dans le secteur du textile et des vêtements sont restés largement supérieurs à ceux pratiqués dans d'autres secteurs industriels. Ces droits de douane, qui ne sont pas touchés par l'ATV, restent donc un outil potentiel pour contrer les effets de la levée des quotas. Pris à la gorge par la concurrence chinoise, de nombreux pays en développement espèrent pouvoir bénéficier de droits de douane "préférentiels " pour pénétrer les marchés européens ou américains.

\subsubsection{Guerre commerciale annoncée}

L'onde de choc s'annonce dévastatrice pour nombre de petits pays producteurs du Sud $^{3}$. Le Bangladesh ou le Cambodge, dont le textile et l'habillement représentent plus des trois quarts des exportations, sont en première ligne. Globalement, entre,

3 Natacha David, «La fin des quotas textiles», Enjeux internationaux, n ${ }^{\mathrm{o}} 7,1^{\mathrm{er}}$ trimestre 2005. 
d'un côté, la Chine, suivie de l'Inde et du Pakistan, et, de l'autre côté, de nombreux pays pauvres d'Asie, d'Afrique et d'Amérique centrale, une guerre commerciale inégale est amorcée. Des millions de pertes d'emplois sont annoncées et les plus touchées seront les travailleuses non qualifiées. Quant aux emplois qui subsisteront, ils risquent de faire les frais du dumping social pratiqué par le géant chinois, où les organisations syndicales indépendantes sont réprimées. Dans ce climat, les Philippines ou le Bangladesh, par exemple, ont déjà annoncé qu'ils allaient revoir leurs règlements de travail à la baisse, alors que les employeurs locaux menacent de délocaliser vers la Chine.

D'autres pays, plus proches de l'Union européenne, vont également sentir les répercussions de la levée des quotas, notamment le Maroc, la Tunisie, la Turquie, la Bulgarie et la Roumanie. Des effets aussi désastreux en termes d'emplois y sont à craindre, avec les problèmes de stabilité et de migration. Ces pays limitrophes vont tenter de jouer la carte de la «montée en gamme», ce qui va exacerber la concurrence avec la production européenne de produits à haute valeur ajoutée. C'est précisément la carte jouée par la Suisse, qui n'a jamais introduit de quotas sur les importations de textiles.

\subsubsection{Arrivée de la Chine}

Depuis l'entrée en vigueur de l'Accord sur les textiles et les vêtements, le marché du textile s'est encore tendu lorsque la Chine est devenue membre de l'OMC en 2001. Selon la Banque mondiale, la Chine, qui, depuis son adhésion, assurait déjà près du quart des exportations mondiales dans l'habillement, devrait en contrôler la moitié d'ici à 2010. A terme, la Banque mondiale prévoit l'extinction de l'industrie textile dans l'Union européenne et aux Etats-Unis. Entre-temps, ces deux puissances ont recouru à la clause de sauvegarde prévue par l'OMC, en cas de déstabilisation trop importante de leurs marchés respectifs. Le Brésil, l'Argentine et la Colombie ont également déjà mis en œuvre ce mécanisme.

L'Union européenne et la Chine ${ }^{4}$ ont signé le 5 septembre 2005 un accord qui a permis de débloquer près de 80 millions de pièces de vêtements chinois importés. Cet accord a ainsi mis fin à ce différend, en accroissant la quantité des exportations chinoises autorisée en 2005. L'accord amende effectivement les termes de l'accord du 10 juin 2005, qui limitait dix types d'exportations de textiles chinois dans l'Union européenne à des hausses annuelles de maximum $8 \%$ à $12,5 \%$ au cours des trois prochaines années. Les vêtements interdits, dont la plupart avaient été commandés avant l'imposition des restrictions à l'importation, ont été bloqués dans les ports européens, après que les contingents convenus en juin 2005 eurent été remplis. Aux fins de l'accord de septembre 2005, les contingents d'importations pour 2005 ont été rehaussés pour permettre de libérer la marchandise bloquée. La Chine a convenu de décompter la moitié de cette hausse au titre des contingents d'importations de 2006, alors que l'Union européenne a convenu d'autoriser l'importation du reste en excès des quantités anté-

4 Centre international pour le commerce et le développement durable (ICTSD), «La Chine signe un accord avec l'UE sur les textiles interdits d'accès; négociations américaines bloquées », Passerelles. Synthèse mensuelle sur le commerce et le développement, vol. 4, nº 12, 22 septembre 2005. 
rieurement acceptées. Ce nouvel arrangement implique que la quantité de textiles chinois autorisés dans l'Union européenne en 2006 sera inférieure à celle prévue initialement. Les détaillants britanniques ont averti qu'à long terme cette situation mènerait à des pénuries de vêtements chinois et à des prix probablement plus élevés pour les consommateurs européens.

Dans l'intervalle, le quatrième round de négociations entre les Etats-Unis et la Chine sur un accord bilatéral visant à résoudre leur différend sur les textiles a échoué le $1^{\text {er }}$ septembre 2005, après trois jours de négociations. Des questions clés restent à résoudre (échéance de l'accord, nombre de produits visés, taux de croissance annuel, etc.). Le même jour, le Comité américain de mise en œuvre des accords sur les textiles ${ }^{5}$ a imposé des restrictions sur les importations de soutiens-gorge et de tissus en filaments synthétiques chinois, recourant au mécanisme de sauvegarde transitoire par produit prévu dans l'accord d'accession de la Chine à l'OMC ${ }^{6}$. Il a toutefois différé une décision sur la mise en place de restrictions pour quatre autres types de vêtements. Les Etats-Unis avaient déjà utilisé à d'autres reprises cette clause de sauvegarde, en réintroduisant des quotas sur les robes de chambre, les soutiens-gorge et les textiles tricotés, suite à la progression de $1000 \%$ de ces importations, dont les quotas avaient été levés en 2002.

\subsection{Organisation mondiale du commerce (OMC)}

L'accord conclu par les pays membres de l'OMC à Genève fin juillet 2004 était censé redonner l'impulsion nécessaire au cycle de Doha, bloqué depuis l'échec de la Conférence ministérielle de Cancùn en septembre 2003. En réalité, les pourparlers ont piétiné pendant la première partie de l'année 2005, obligeant à une révision du calendrier. La contrainte du fast track ${ }^{7}$ américain a donné un caractère décisif à la Conférence ministérielle de Hongkong et a intensifié les discussions dès l'automne 2005. Entré en fonction le $1^{\text {er }}$ septembre 2005, le nouveau directeur général, Pascal Lamy, n'a pas ménagé ses efforts pour redynamiser le processus. Sans grand succès cependant, au point que les ambitions de Hongkong ont été rapidement revues à la baisse.

[Da Annuaire 2005, $\mathrm{n}^{\circ}$ 1, accord-cadre de juillet 2004, pp. 78-82.

\subsubsection{L'année 2005: état des négociations}

En 2005, les négociations ${ }^{8}$ ont buté sur la compréhension de ce qu'est ou devrait être un «cycle du développement». Pour les pays industrialisés, certains pays

5 US Committee for the Implementation of Textile Agreements (CITA). Cet organe gouvernemental gère le commerce des textiles des Etats-Unis.

6 OMC, Protocole d'accession de la République populaire de Chine à l'OMC, article 16, 10 novembre 2001.

7 Le fast track est l'autorisation de négocier et conclure des accords commerciaux accordée par le Congrès américain à l'administration. Il expire mi-2007 et nombre d'observateurs estiment qu'il sera très difficile à George Bush d'obtenir une prolongation de cette procédure devant un parlement qui s'affiche de plus en plus protectionniste. Il est donc impératif pour l'OMC de conclure le cycle de Doha avant cette date.

8 Pour le détail des dossiers de l'OMC durant l'année 2005, voir ICTSD, Dossiers sur le cycle de Doha. Mise à jour de Hongkong, vol. 3, novembre 2005. 
émergents ainsi que les représentants de l'économie privée, cela passe avant tout par une libéralisation des marchés et la croissance économique supposée en découler. Pour d'autres, comme certains pays en développement et une grande part des ONG, cela suppose l'octroi ou le maintien d'un vrai «espace politique» (policy space), qui va au-delà du traitement spécial et différencié ${ }^{9}$ octroyé par l’OMC (voir encadré «Espace politique» page suivante).

\section{$\square$ Cycle du développement?}

Le cycle lancé à Doha en 2001 avait pour but de mettre les intérêts des pays en développement au cœur des négociations, d'honorer les promesses qui leur avaient été faites pendant le cycle de l'Uruguay, de corriger les manques, déséquilibres et inégalités structurels des accords existants, qui leur sont défavorables. C'est ce que les pays en développement ont rappelé à deux reprises juste avant la Conférence ministérielle de Hongkong ${ }^{10}$. Quatre éléments concernant le cycle du développement ressortent de la Déclaration de Doha: la résolution de toute une série de questions de mise en ouvre; la fin de pratiques jugées déloyales (soutiens internes, subventions à l'exportation et mesures protectionnistes) qui permettent aux pays du Nord de défendre leurs marchés agricoles tout en ruinant ceux du Sud; une meilleure réglementation du traitement spécial et différencié, en particulier pour les pays les moins avancés; un renforcement $\mathrm{du}$ droit des Etats membres à utiliser des licences obligatoires et à importer, pour des raisons de santé publique, des versions génériques de médicaments protégés par des brevets.

En «vendant» un «cycle du développement» aux pays du Sud, les pays industrialisés ont, de facto, ouvert la boîte de Pandore de la gouvernance de l'économie mondiale et du rôle que doit y jouer l'OMC. Avec ces trois questions de fond:

口 Dans quel but: maximiser les libéralisations ou encadrer les marchés?

口 Avec quel «espace politique» pour les pays?

๖ Selon quels objectifs de développement (sécurité et souveraineté alimentaire, approvisionnement en eau, éducation et santé de base, protection de l'environnement, etc.) et quelles préférences collectives (économiques, sociales, écologiques)?

Ces questions excèdent le cadre strict de l'OMC et sont en tension avec l'établissement de règles uniformes pour tous. Elles devraient pourtant être sousjacentes à toutes les négociations commerciales. De nombreux économistes contestent de plus en plus la corrélation entre libéralisation des marchés, croissance économique et développement. Dans cette ligne, Pascal Lamy a, peu avant son accession à la direction générale de l'OMC, appelé à un nouveau «consensus»: «Ce qui est épuisé, c'est l'idée que la libéralisation, l’ouverture

9 Les accords de 1'OMC contiennent des dispositions spéciales, appelées «traitement spécial et différencié », qui confèrent des droits particuliers aux pays en développement. Il s'agit par exemple de dispositions prévoyant des délais plus longs pour mettre en œuvre les accords et les engagements.

10 La Conférence des ministres du Commerce de l'Union africaine a adopté, le 24 novembre 2005, une déclaration intitulée The Arusha Development Benchmarks. Ensuite, le 28 novembre 2005, une soumission, intitulée Reclaiming Development in the WTO Doha Development Round, a été faite au Comité sur le commerce et le développement par neuf pays: Afrique du Sud, Argentine, Brésil, Inde, Indonésie, Namibie, Pakistan, Philippines et Venezuela. 
et la privatisation forment une recette miracle et suffisante. Aujourd'hui, on ne peut plus croire que les vertus de la théorie économique finissent nécessairement par s'incarner dans la réalité sociale sans qu'il soit nécessaire d'y ajouter quoi que ce soit. L'heure est venue de bâtir le "consensus de Genève", qui ne remet pas en cause l'idée fondamentale qu'il y a un gain à l'ouverture des échanges pour ceux qui y participent, mais qui s'attache aux conditions dans lesquelles les vertus théoriques de ce gain peuvent se traduire par du pouvoir d'achat, de la croissance, de la lutte contre la pauvreté. ${ }^{11}$

\section{Espace politique}

Le concept d'espace politique (policy space) désigne la marge de manœuvre des gouvernements pour mener des politiques économiques qui correspondent à leur situation spécifique et aux besoins réels de leurs populations. Cela en recourant à un choix d'instruments et à des flexibilités qui vont plus loin que ceux autorisés par les organisations internationales comme les institutions de Bretton Woods et I'OMC. Cette notion figure dans la Déclaration de la CNUCED Xla, qui s'est tenue à São Paulo du 13 au 18 juin 2004; c'était la première fois qu'elle était reconnue dans un texte intergouvernemental. Dans le Consensus de São Paulo, il est stipulé au paragraphe 8: "Eu égard aux objectifs de développement, il est particulièrement important pour les pays en développement que tous les pays prennent en compte la nécessité de concilier au mieux marge d'action nationale [traduction de l'original anglais policy space] et disciplines et engagements internationaux $»^{b}$.

[D] Annuaire 2005, $\mathrm{n}^{0} 1,11^{\mathrm{e}}$ Conférence quadriennale de la CNUCED, pp. 85-88.

L'une des questions clés liées à cet espace politique est de savoir qui détermine les politiques de l'Etat, selon quels intérêts et au profit de qui. Etant donné le manque de légitimité démocratique et/ou de représentativité de nombre de gouvernements qui négocient à I'OMC, il ne suffit donc pas de redonner à ceux-ci la fonction de régulation, de contrôle et de redistribution qui leur revient, mais de créer, au sein même des Etats et à I'OMC, des structures et procédures démocratiques de consultation et de décision, qui permettent aux populations de se faire entendre (voir plus bas section 6.5.2).

a CNUCED, L'esprit de São Paulo, doc. TD/L.382, 2004.

b CNUCED, Consensus de São Paulo, doc. TD/410, 2004.

Voici en détail l'état des négociations en 2005 des principaux dossiers de l'OMC: l'agriculture, les biens industriels, les services et la propriété intellectuelle.

\section{$\square$ Agriculture}

L'accès des pays en développement aux marchés agricoles ${ }^{12}$ des pays industrialisés est au cœur des débats. Comme l'a répété à plusieurs reprises le ministre brésilien du Commerce, c'est «le moteur des négociations». L'agriculture est en effet le secteur où les inégalités structurelles sont les plus grandes entre pays industrialisés et pays en développement. Pour les pays agro-exportateurs du Sud, réunis dans le G-20 $0^{13}$, un «cycle du développement» digne de ce nom supposerait que les pays riches acceptent des ouvertures dans le domaine agricole,

11 Pascal Lamy cité par Thierry Pech et Wojtek Kalinowski, «Politiser la mondialisation. Entretien avec Pascal Lamy», La Vie des idées, juin 2005.

12 OMC, page «agriculture» : <www.wto.org/indexfr.htm > >domaines > marchandises >agriculture.

13 Le G-20, qui demande une ouverture accrue des marchés du Nord, comprend en réalité 21 membres : Afrique du Sud, Argentine, Bolivie, Brésil, Chili, Chine, Cuba, Egypte, Guatemala, Inde, Indonésie, Mexique, Nigeria, Pakistan, Paraguay, Philippines, Tanzanie, Thaïlande, Uruguay, Venezuela et Zimbabwe. Il représente plus de la moitié de la paysannerie mondiale. 
sans exiger des concessions massives en retour dans d'autres domaines. Ce que refusent les pays industrialisés, qui ne sont prêts à considérer d'éventuelles ouvertures dans l'agriculture qu'en contrepartie d'un meilleur accès de leurs biens industriels et services aux marchés des pays en développement. Le ministre indien du Commerce a vivement critiqué cette attitude et les demandes «exagérées » adressées aux pays en développement, accusant les pays industrialisés d'avoir transformé le «cycle du développement» de Doha en «cycle de l'accès au marché».

Les positions se sont précisées et affirmées dès octobre 2005, sous une forme d'un «jeu de la faute» (blame game), où chaque partie faisait monter les enchères en cherchant à rendre les autres responsables d'un éventuel échec des négociations. Les Etats-Unis et le G-20 veulent plus d'ouverture des marchés de la part de 1'Union européenne et du G-10 ${ }^{14}$. Le G-20 estime que les propositions de réduction des soutiens internes des Etats-Unis et de l'Union européenne sont non seulement décevantes, mais aussi trompeuses, car sans effet réel, étant donné les tours de passe-passe possibles (transferts de subventions d'une catégorie ${ }^{15}$ à l'autre). Avec le G-33 $3^{16}$ et le groupe des pays pauvres d'Afrique, des Caraïbes et du Pacifique (ACP), il estime que les aspects de traitement spécial et différencié ne sont pas suffisamment pris en compte, notamment les questions de sécurité alimentaire et de développement rural (produits spéciaux et mesures de sauvegarde). Les pays ACP, qui, pour la première fois, ont élaboré leur propre position ${ }^{17}$, pensent que les demandes d'ouverture des grands exportateurs agricoles auront des effets dévastateurs sur leurs agricultures et exigent plus d'attention au problème de l'érosion de leurs préférences. La Suisse les soutient et a loué leur initiative.

La marge de manœuvre des négociateurs est limitée. Le commissaire européen au Commerce a subi les foudres du gouvernement français, qui a estimé qu'il avait outrepassé son mandat. Côté suisse, soumis aux pressions du lobby agri$\operatorname{cole}^{18}$, le Secrétariat d'Etat à l'économie (seco) a qualifié certaines des propositions agricoles sur la table de l'OMC d'inacceptables. Celles-ci demandaient en particulier la baisse et le plafonnement des tarifs, ainsi que la limitation du nombre de «produits sensibles » bénéficiant de flexibilités spéciales pour soutenir le rôle multifonctionnel de l'agriculture. Tant le président français que le Conseil fédéral ont menacé d'user de leur «droit de veto», au cas où les libéralisations agricoles excéderaient par trop le seuil du supportable, ainsi que le cadre de leur politique agricole.

14 Dirigé par la Suisse, le G-10 est le groupe des pays industrialisés importateurs nets de produits agricoles, qui demandent une forte protection de leur agriculture: Bulgarie, Corée du Sud, île Maurice, Islande, Israël, Japon, Liechtenstein, Norvège, Suisse et Taïwan.

15 Sur les catégories de soutien interne, voir le glossaire lié aux négociations sur l'agriculture dans Annuaire 2004, $\mathrm{n}^{\circ}$ 1, p. 90.

16 Coordonné notamment par l'Indonésie, le G-33 est une alliance d'une quarantaine de pays en développement, qui défendent les principes de la sécurité alimentaire et du droit au développement rural.

17 Les pays ACP ont, le 21 octobre 2005, lors d'une réunion informelle de la session spéciale du Comité sur l'agriculture, fait une proposition sur l'accès aux marchés qui mettait en évidence la vulnérabilité de nombreux pays en développement face à l'ouverture illimitée des marchés.

18 L'Union suisse des paysans (USP) a notamment organisé une importante journée de mobilisation le 17 novembre 2005, où 10'000 paysans ont marché dans les rues de Berne pour exprimer leur peur de disparaître. 


\section{$\square$ Biens industriels}

Avec le retrait des thèmes dits de Singapour ${ }^{19}$, la libéralisation du commerce des biens industriels ${ }^{20}$ a repris une place déterminante dans les négociations. Les Etats-Unis et l'Union européenne, de même que la Suisse, ont fait des demandes importantes aux pays en développement:

- consolidation (plafonnement) de la quasi-totalité de leurs tarifs;

$\checkmark$ diminution drastique des droits de douane selon la formule dite «suisse $»^{21}$.

Ces mesures feraient perdre non seulement d'importantes sources de revenus budgétaires aux pays en développement, mais aussi une grande part de leur «espace politique». L'Union africaine, les pays ACP et les pays les moins avancés (PMA) ont qualifié ces demandes de «menace majeure pour leur développement». Dans la mesure où leurs tarifs douaniers sont en moyenne nettement plus élevés que ceux des pays industrialisés, l'engagement des pays en développement serait de facto beaucoup plus important. Un résultat qui serait contraire au mandat de Doha qui, centré théoriquement sur les «intérêts et besoins spéciaux» des pays en développement, prévoit «moins qu'une pleine réciprocité» en leur faveur.

\section{$\square$ Services}

Dans le domaine des services ${ }^{22}$, tous les pays membres de l'OMC ont dû remettre leurs propositions et demandes de libéralisation jusqu'à fin juin 2005. La plupart l'ont fait, mais les pays industrialisés estiment que les offres des pays en développement sont insuffisantes. La Suisse a développé une méthode de mesure qu'elle a transmise à l'OMC, permettant d'évaluer et de comparer les offres grâce à une formule unique ${ }^{23}$. L'Union européenne a pour sa part proposé un système exigeant un engagement minimum - tant par rapport au nombre de secteurs à ouvrir qu'au degré de libéralisation - de la part de tous les pays (benchmarking). Ainsi, les pays industrialisés devraient prendre de nouveaux engagements ou améliorer leur offre dans 139 des 163 sous-secteurs recensés par l'OMC, et les pays en développement dans 93.

L'objectif des pays industrialisés, dont la Suisse, est de permettre l'ouverture des marchés des services financiers et des assurances, des transports, de l'énergie, des télécommunications et du tourisme, au profit des prestataires étrangers. En contrepartie, les demandes récurrentes de certains pays émergents visant un meilleur accès aux marchés du travail des pays riches (mode 4$)^{24} \mathrm{n}$ 'ont toujours pas rencontré d'écho positif. De nombreux pays en développement ont rejeté le

19 En juillet 2004, sous la pression des pays en développement, les sujets liés aux investissements, à la compétition et à la transparence des marchés publics ont été enlevés de l'agenda de l'OMC.

20 OMC, page «accès aux marchés »: <www.wto.org/indexfr.htm> >domaines >marchandises >accès aux marchés pour les marchandises.

${ }^{21}$ La formule dite «suisse» s'applique à chaque ligne de produits et implique une baisse plus forte des tarifs les plus élevés, ainsi que l'élimination rapide des droits de douane dans certains secteurs comme les produits de la pêche, les pierres et métaux précieux, etc.

22 OMC, page « services »: <www.wto.org/indexfr.htm> >domaines >services.

23 Methodology Developed by Switzerland to Assess Schedules of Commitments, working document by Switzerland, Bern, 13 septembre 2005.

24 Le mode 4 couvre le mouvement temporaire de personnes physiques, où le fournisseur du service passe la frontière sous forme d'un déplacement physique pour une période limitée. 
système de l'engagement minimal, estimant notamment que cela conduisait à changer la nature même de l'Accord général sur le commerce des services (AGCS), qui laisse en principe à chaque pays le choix de procéder à des libéralisations progressives, dans une mesure adaptée à sa situation et ses intérêts.

\section{$\square$ Propriété intellectuelle}

Afin de donner un signal positif aux pays en développement, l'OMC a approuvé début novembre 2005 un amendement ${ }^{25}$ à l'Accord sur les aspects des droits de propriété intellectuelle qui touchent au commerce (ADPIC/TRIPs) ${ }^{26}$. Juste avant Cancun, en août 2003, elle avait autorisé d'une manière temporaire les pays en développement sans capacités de production à utiliser des licences obligatoires et à importer, pour des raisons de santé publique, des versions génériques de médicaments protégés par des brevets. Cette décision a été transformée en «solution permanente», assorties de diverses conditions (définition, notification, étiquetage, etc.) visant à éviter la libre circulation et la réexportation ainsi qu'une pression sur les prix.

La Suisse, qui fait partie avec les Etats-Unis des pays les plus restrictifs sur cette question, a estimé «pouvoir vivre avec ce consensus» ${ }^{27}$. Cette mesure a par contre été critiquée par de nombreuses organisations de la société civile, qui estiment que les conditions et procédures prévues sont si lourdes et complexes qu'elles rendent sa mise en œuvre quasi impossible. A cet égard, on peut noter que la dérogation adoptée en août 2003 n'a pas été utilisée.

Par ailleurs, l'OMC a accordé au groupe des pays les moins avancés un délai supplémentaire de sept ans et demi - soit jusqu' au 1 1er juillet 2013 - pour mettre en œuvre les dispositions de l'Accord sur les ADPIC; les pays en question avaient demandé une extension de quinze ans.

\subsubsection{Les dix ans de l'OMC}

L'OMC a fêté en 2005 son dixième anniversaire ${ }^{28}$. Les différentes manifestations organisées à cette occasion ont permis de montrer que les blocages dont souffre l'OMC ne sont pas seulement le fruit de conflits d'intérêts ou de conceptions divergentes sur ce qu'est un «cycle du développement», mais qu'ils sont aussi le reflet d'un certain nombre de mutations plus fondamentales: la nouvelle donne géopolitique avec la montée en puissance des pays en développement, la complexification des jeux d'alliance au-delà des clivages NordSud, ainsi que les grippages institutionnels (voir plus haut section 6.3.1).

$25 \mathrm{OMC}$, Les pays les plus pauvres auront plus de temps pour appliquer les règles de propriété intellectuelle, communiqué de presse, $\mathrm{n}^{\circ} 424,29$ novembre 2005.

26 OMC, page «propriété intellectuelle»: <www.wto.org/indexfr.htm> >domaines >propriété intellectuelle.

27 Propos de Luzius Wasescha (seco), cités par Ram Etwareea, «L'accord sur les médicaments, de bon augure pour Hongkong », Le Temps, 7 décembre 2005.

28 OMC, page «dixième anniversaire»: <www.wto.org/indexfr.htm> >1'OMC >10 e anniversaire de l'OMC. 


\section{$\square$ Montée en puissance des pays en développement}

L'un des principaux enseignements de 2005 est que l'on se trouve en face d'une redistribution des cartes dans la géopolitique du commerce mondial, qui se manifeste notamment par la montée en force des pays en développement, emmenés par les puissances commerciales émergentes comme le Brésil, l'Inde et l'Afrique du Sud. Conscients de leur poids croissant dans le commerce mondial, comprenant toujours mieux les rouages de la machine OMC, ces pays exigent de plus en plus d'être entendus. Ils ont appris à s'unir et à s'organiser en diverses alliances stratégiques, sur une base dorénavant plus pragmatique qu'idéologique :

๑ dans le G-20 pour l'ouverture des marchés agricoles du Nord;

$\checkmark$ dans le G-33 pour des mesures de sécurité alimentaire;

$\checkmark$ dans le G- $90^{29}$, hier contre les thèmes de Singapour, aujourd'hui en faveur d'un round for free ${ }^{30}$.

L'autre fait significatif est que, malgré l'hétérogénéité et les intérêts parfois divergents de leurs membres, ces blocs ont résisté aux pressions et chantages des Américains et des Européens qui ont tout fait pour les diviser, en particulier en conviant l'Inde et le Brésil à la table des décideurs ${ }^{31}$. En septembre 2005, à Bhurban (Pakistan), le G-20 a resserré ses rangs en affirmant avec force sa volonté de renforcer ses alliances avec les autres groupes de pays en développement en prenant en compte leurs préoccupations. Le point de convergence est la réalisation de la dimension «développement» du cycle de Doha.

\section{$\square$ Complexification des intérêts}

Un autre changement est la complexification des négociations et des jeux d'alliance. L'OMC ne peut plus être réduite à une seule ligne de clivage Nord-Sud. Au sein des blocs, les intérêts divergent et les pouvoirs de négociation varient énormément. L'OMC est devenue un monde multipolaire, où cohabitent plusieurs Nords (les Etats-Unis, l'Union européenne, le G-10) et plusieurs Suds (Brésil très libre-échangiste, Inde plutôt protectionniste, pays africains soucieux de préserver leurs accès préférentiels aux marchés du Nord, etc.). Cette hétérogénéité est d'ailleurs l'une des raisons qui expliquent le choix de l'ancien commissaire européen au Commerce, Pascal Lamy, à la tête de l'OMC, contre trois candidats du Sud. On n'a pas assisté cette fois à la bataille Nord-Sud qui avait marqué l'élection précédente et abouti à un mandat partagé entre le Néo-Zélandais Mike Moore et le Thaïlandais Supachai Panitchpakdi. Habile diplomate, Lamy a pris pour adjoints trois ressortissants du Sud (Chili, Rwanda, Inde) et un Américain.

\section{$\square$ Blocage institutionnel}

Le troisième effet de cette nouvelle donne est d'ordre institutionnel. Le temps est révolu où quelques pays nantis pouvaient faire la loi. Il faut maintenant tenir

29 Le G-90 représente les pays ACP et les pays les moins avancés.

30 Un round for free est un cycle de négociations qui n'exige aucune concession de la part des pays en développement.

31 Par exemple, l'accord-cadre de juillet 2004 a été concocté par les FIPs (five interested parties) regroupant les Etats-Unis, l’Union européenne, le Brésil, l'Inde et l'Australie. 
compte des pays en développement - $80 \%$ des membres. Ces derniers sont parfois soutenus dans leur lutte par des ONG de mieux en mieux informées et réactives $^{32}$. Quelles procédures de consultation et de décision instaurer pour garantir le caractère représentatif des négociations, sans pour autant créer des situations de blocage? Comment arriver à des accords par consensus avec 148 membres, qui seront plus de 170 dans quelques années, en tenant compte de toutes les attentes et spécificités? Comment échapper à la paralysie qui menace le système?

Le directeur général de l'OMC, Pascal Lamy, s'est promis de réformer l'organisation, qu'il avait qualifiée de «structure médiévale» après l'échec de Cancùn. De nombreuses propositions ${ }^{33}$ sont sur la table de l'OMC, élaborées par un comité de huit membres, sous la direction de l'ex-directeur du GATT Peter Sutherland. Cet ensemble de recommandations évoque notamment :

$\checkmark$ le renforcement du secrétariat;

- l'accroissement des pouvoirs du directeur général;

$\checkmark$ un système à deux vitesses, avec des accords plurilatéraux n'engageant que les membres qui le désirent;

$\checkmark$ la restriction du droit de veto;

$\checkmark$ la création d'un conseil permanent représentatif, etc.

Un signe du pouvoir accru du secrétariat est le rôle pris par Pascal Lamy dans la préparation de la Conférence ministérielle de Hongkong. C'est la première fois que le directeur général rédige lui-même le projet de déclaration ministérielle, un rôle jusqu'ici dévolu au président de la conférence.

\subsection{Conférence ministérielle de Hongkong}

En prenant ses fonctions, le nouveau directeur général, Pascal Lamy, avait déclaré que la Conférence ministérielle de Hongkong devrait permettre d'accomplir les deux tiers du chemin vers la conclusion du cycle de Doha ${ }^{34}$. Les négociations demeurant pour l'essentiel bloquées sur les principaux dossiers, il a dû cependant revoir rapidement cet objectif à la baisse. Un «recalibrage» d'autant plus nécessaire qu'après le fiasco de Cancùn, il fallait à tout prix éviter un nouvel échec qui risquait non seulement de fragiliser institutionnellement l'OMC, mais aussi de démotiver les Etats membres. On a donc décidé que cette conférence servirait avant tout à tenter de résoudre certaines impasses, progresser sur quelques points spécifiques et définir une feuille de route claire pour la suite du processus.

32 On l'a vu avec les cas récents du coton africain, des thèmes de Singapour ou encore de certaines revendications liées à la sécurité alimentaire, où les pays en développement ont travaillé en étroite collaboration avec certaines ONG comme Oxfam International, Third World Network, Focus on the Global South, le Centre international pour le commerce et le développement durable (ICTSD), IDEAS Centre, etc.

33 Conseil consultatif de l'OMC, L'avenir de l'OMC. Relever les défis institutionnels du nouveau millénaire, rapport du Conseil consultatif à l'ancien directeur général, Supachai Panitchpakdi, Genève, 2004.

34 Objectif annoncé le 14 septembre 2005 au Comité sur les négociations commerciales et à son premier briefing aux médias le même jour. 


\subsubsection{Position suisse}

Dans cette situation, le Conseil fédéral n'a pas revu son mandat de négociation adopté pour Cancùn, se réservant la possibilité d'organiser, en cas de besoin, une consultation téléphonique entre ses membres pendant la Ministérielle. Sa position de fond n'a pas changé: libéralisations ambitieuses dans les domaines des services et des biens industriels, ouvertures agricoles limitées dans le respect des considérations non commerciales et la «conciliation des intérêts des pays exportateurs et importateurs $»^{35}$. Il se prononce également en faveur de règles commerciales améliorées et d'une nouvelle catégorisation des pays en développement. Le conseiller fédéral Joseph Deiss, qui dirigeait la délégation suisse, a déclaré dans son discours en plénière à l'ouverture de la Conférence ministérielle de Hongkong: «Personne n'a intérêt à l'échec des négociations. En effet, l'histoire a démontré que l'ouverture à l'échange stimule la croissance. [...] Aujourd'hui, la réflexion doit porter sur les moyens d'en faire profiter le plus grand nombre. ${ }^{36}$

Début décembre, economiesuisse s'en est prise au gouvernement, l'accusant de pratiquer «un protectionnisme agricole excessif et dépassé», qui ne reflète pas les réalités et priorités économiques de la Suisse ${ }^{37}$. L'association patronale a demandé au Conseil fédéral de «ne pas freiner les négociations» et de «défendre les intérêts offensifs majeurs» de l'économie dans les marchés des services et des biens industriels.

De son côté, la Coordination Suisse-OMC ${ }^{38}$, qui réunit des syndicats, associations paysannes et ONG de développement et d'environnement, a demandé au Conseil fédéral de «s'engager pour une réorientation du cycle de négociations en cours», trop axé sur l'accès au marché. Pour cela, «la Suisse devrait renoncer à ses demandes de libéralisation exagérées dans les domaines de l'industrie et des services, car les pays pauvres auraient plus à perdre qu'à gagner ${ }^{39}$.

\section{$\square$ Principaux résultats}

La Conférence ministérielle a été marquée par des discussions souvent laborieuses et tendues. Les Etats-Unis et le G-20 ont exercé une pression très forte sur l'Union européenne, l'accusant de bloquer les négociations par son refus de nouvelles concessions sur l'agriculture. En termes géopolitiques, on a assisté à de nouvelles manifestations de solidarité entre pays du Sud. Le G-20, le G-33, les pays ACP et les PMA ont, pour la première fois dans l'histoire de l'OMC, tenu une conférence de presse ensemble et présenté une position commune. Une alliance qui doit cependant être relativisée, des intérêts divergents entre pays en

35 Formule reprise par le conseiller fédéral Joseph Deiss dans son intervention en plénière lors de la Conférence ministérielle de l'OMC à Hongkong. OMC, Déclaration de S.E.M Joseph Deiss, conseiller fédéral, chef du Département fédéral de l'économie, doc. WT/MIN(05)/ST/2, 15 décembre 2005.

36 Ibid.

37 Economiesuisse, L'économie suisse favorable à une stratégie offensive pour la Conférence ministérielle de l'OMC, communiqué de presse, 30 novembre 2005.

38 Les membres du comité de la Coordination Suisse-OMC sont: Alliance Sud, Déclaration de Berne, Pro Natura, Union suisse des paysans, Union syndicale suisse, Uniterre.

39 Coordination Suisse-OMC, La Coordination Suisse-OMC exige un vrai cycle du développement, communiqué de presse, 5 décembre 2005. 
développement se manifestant dans les différentes green rooms ${ }^{40}$ qui ont émaillé la semaine.

En termes de contenu, aucun Etat-membre de l'OMC ne voulant être tenu responsable d'un échec, Hongkong a abouti à une déclaration ministérielle ${ }^{41}$. Celleci a, dans l'ensemble, réaffirmé les principaux éléments de l'accord conclu en juillet 2004, qui servait de cadre aux négociations. Les principaux éléments, déterminants pour la suite des négociations, sont les suivants:

- agriculture: malgré les réticences de l'Union européenne et du G-10, une date butoir a été fixée à 2013 pour toutes les formes de subvention aux exportations. Le besoin des pays en développement a été réaffirmé de pouvoir désigner un certain nombre de produits spéciaux liés à la sécurité alimentaire et échappant aux réductions tarifaires;

- coton: peu de progrès ont été réalisés sur la problématique du coton, thème à nouveau très médiatisé. Bien que plus ouverts aux revendications des pays africains (Bénin, Burkina Faso, Mali et Tchad), les Etats-Unis sont pour l'essentiel restés intransigeants. La question sera traitée dans le cadre de l'Accord sur l'agriculture. Si les subventions des pays développés à l'exportation de coton devront être éliminées d'ici à fin 2006, rien n'a été décidé pour les soutiens internes, qui constituent la principale source de distorsion des échanges et de dumping en faisant chuter les prix sur le marché mondial;

๖ biens industriels: le principe d'une formule dite «suisse» a été adopté pour les réductions tarifaires - avec divers coefficients selon le degré de développement des pays - ainsi que le lancement d'initiatives de libéralisation accrue et rapide dans certains secteurs;

- services: un cadre de négociations ${ }^{42}$ a été accepté, malgré de fortes réticences du G-90 qui contestait son contenu, trop favorable aux pays industrialisés, et la manière peu transparente et démocratique dont il avait été défini. Le compromis rend, de facto, possibles des pressions accrues pour des engagements plus poussés et contraignants en matière d'ouverture des marchés, ainsi que le lancement de négociations plurilatérales ${ }^{43}$;

- traitement spécial et différencié: sur les cinq propositions en discussion, issues des PMA, une seule a fait l'objet de débats nourris et de propositions concrètes. Les pays développés et en transition devront, jusqu'en 2008, offrir un accès libre (sans droits de douane ni quotas) aux produits venant des PMA. Ils auront cependant la possibilité d'exclure de ces préférences jusqu'à $3 \%$ des produits. En d'autres termes, par exemple, les Etats-Unis pourront continuer à protéger leur industrie textile contre les importations du Bangladesh. Ce «paquet du développement» contient également des promesses pour des programmes d'aide technique et financière liée au commerce.

40 Réunions «informelles» de négociations qui rassemblent un nombre limité de pays (en général une trentaine) plus ou moins représentatifs des divers intérêts et positions en présence.

41 OMC, Déclaration ministérielle adoptée le 18 décembre 2005, doc. WT/MIN(05)/DEC, 2005.

42 OMC, Déclaration ministérielle, op. cit., annexe C.

43 Dans le jargon de l'OMC, le «plurilatéralisme» qualifie les engagements souscrits par certains membres seulement, dans l'idée de faire avancer les choses autour d'un noyau dur de pays en attendant que les autres prennent le train en marche. 
Les Etats membres ont reprécisé le calendrier des négociations, dans l'optique de conclure le cycle de Doha à fin 2006. Ils ont fixé au 30 avril 2006 la date limite pour l'établissement de modalités et au 31 juillet 2006 celle pour les premières propositions concrètes. La possibilité d'une nouvelle conférence ministérielle (au printemps à Genève) a été évoquée, mais pas confirmée.

\section{$\square$ Réactions}

Le conseiller fédéral Joseph Deiss a salué le «succès» de Hongkong, lors d'une conférence de presse à Hongkong ${ }^{44}$. Il a estimé que la déclaration ministérielle constituait «une excellente base pour le travail futur dans le cycle de Doha. Les décisions prises ne font pas qu'enregistrer les progrès accomplis à Genève, mais contiennent de nombreux ajouts, parfois sur des questions sensibles». Il a salué plusieurs avancées, notamment, dans le traitement des «produits agricoles sensibles » pour les pays développés, l'élimination des soutiens aux exportations en 2013. Les textes sur les biens industriels et les services sont «satisfaisants», même si le «niveau d'ambition doit encore être précisé». Par contre, il a regretté le peu d'importance accordé à la protection étendue des indications géographiques et aurait souhaité plus de générosité pour le «paquet du développement», qui aurait dû couvrir la totalité des produits des PMA.

Economiesuisse a déploré les «progrès minimaux» de la conférence, en particulier dans les «domaines décisifs comme l'accès au marché pour les biens industriels, l'ouverture du commerce des services et la facilitation du commerce ${ }^{45}$.

Les ONG suisses présentes à Hongkong (Alliance Sud, Déclaration de Berne, Pro Natura) se sont déclarées «déçues et inquiètes» des résultats de la Ministérielle, qui présentent en réalité «peu d'avancées vers un authentique cycle du développement ${ }^{46}$. La date butoir pour l'élimination des subventions à l'exportation a été repoussée de 2010 à 2013, le «paquet du développement» est un «leurre ${ }^{47}$, le problème des cotonniers africains reste entier, les «pays industrialisés - dont la Suisse - ont pu renforcer les dispositions vers une libéralisation accrue des biens industriels et des services ».

\subsection{Activités des organisations non gouvernementales de développement}

Les activités des organisations non gouvernementales de développement concernant les relations commerciales internationales ont été novatrices en 2005. Trois sortent particulièrement du lot: la Semaine d'action dans le monde entier, la large consultation d'Alliance Sud ${ }^{48}$ auprès d'ONG du Sud ainsi que le colloque de la Déclaration de Berne sur les services financiers.

44 Statement of Federal Councilor Joseph Deiss, Head of Delegation Meeting, Hongkong, 18 December 2005 (prise de position suisse après la réunion finale des chefs de délégation).

45 Economiesuisse, L'OMC n'a fait que des progrès minimaux à Hongkong, communiqué de presse, 19 décembre 2005.

46 Alliance Sud/Déclaration de Berne/Pro Natura, Les ONG suisses déçues et inquiètes, communiqué de presse, 18 décembre 2005.

47 Alliance Sud/Déclaration de Berne/Pro Natura, Le paquet du développement est un leurre, communiqué de presse, 16 décembre 2005.

48 Alliance Sud est le nouveau nom qu'a pris la Communauté de travail des œuvres d'entraide Swissaid/Action de carême/Pain pour le prochain/Helvetas/Caritas/EPER en août 2005. 


\subsubsection{Semaine d'action mondiale sur le commerce}

Plus de 10 millions de personnes, mobilisées dans 80 pays par des centaines d'ONG, mouvements de base et réseaux, ont pris part à la Semaine mondiale d'action sur le commerce ${ }^{49}$. Cette semaine s'est déroulée du 10 au 16 avril 2005, sous le slogan Trade justice not free trade. Il s'agissait de mener, sur une semaine, une campagne internationale de grande envergure à partir de manifestations décentralisées, chacune ayant son contenu et sa forme propres. Le but était triple: démonter le mythe de la libéralisation des marchés et de la privatisation des services publics comme sésames du développement, tels qu'ils sont prônés par le G8, le FMI, la Banque mondiale, l'OMC et nombre de gouvernements, promouvoir des alternatives au système commercial façonné par l'OMC sur le plan multilatéral et par les accords commerciaux bilatéraux et régionaux, ainsi qu'exiger des politiques commerciales qu'elles donnent la priorité aux droits humains, à la réduction de la pauvreté, à la sécurité alimentaire, à la santé et à l'éducation, en soumettant notamment les multinationales à des normes contraignantes.

En Suisse, plus de 50 organisations non gouvernementales ont participé à cette initiative, au travers de nombreuses manifestations aux quatre coins du pays. En Suisse romande, l'accent a été mis sur la campagne «GATS et démocratie», lancée en septembre 2003 par Alliance Sud ${ }^{50}$ auprès des élus locaux et relayée dès 2004 par Attac ${ }^{51}$. Selon les ONG suisses, le Conseil fédéral mène une stratégie «offensive» de libéralisation dans le cadre de l'Accord général sur le commerce des services (AGCS/GATS). Comme l'a révélé la campagne, les autorités cantonales et communales témoignent en fait d'une «confiance quasi aveugle» et d'une «grande allégeance» au Conseil fédéral; elles ont de la peine à évaluer l'impact à moyen et long terme de l'accord. Pour Alliance Sud et Attac, le manque de transparence et le déficit démocratique qui caractérisent ces négociations sur les services sont «inacceptables». Sensibilisés par cette campagne, certains élus en ont pris conscience et ont lancé le débat. Résultat: à l'instar d'un bon millier de municipalités en Europe, plus de 70 communes se sont déclarées «zones hors GATS» en Suisse.

\subsubsection{Consultation auprès d'ONG du Sud sur le commerce international}

A l'occasion des dix ans de l'OMC, Alliance Sud a organisé une vaste consultation des organisations non gouvernementales au Sud. Cinquante-sept ONG, soit près de la moitié des organisations sollicitées - pour un bon nombre, des partenaires des œuvres membres d'Alliance Sud -, ont répondu au questionnaire et une quinzaine de délégués d'Afrique, d'Amérique latine et d'Asie ont participé au symposium, les $1^{\text {er }}$ et 2 juin 2005 à Berne, sur le thème «Quel commerce pour quel développement?».

L'objectif était quadruple: repenser les relations entre commerce et développement, les rôles et compétences de l'OMC, les alternatives possibles, ainsi que le

49 Semaine mondiale d'action sur le commerce: <www.april2005.org $>$.

50 Au nom de la Coordination Suisse-OMC, <www.alliancesud.ch > >thèmes/campagnes >commerce $>$ archive >campagne GATS et démocratie.

51 Pour des détails sur cette campagne et la liste des communes, voir <www.suisse.attac.org $>$. 
contenu d'un authentique «round du développement»; élargir le champ de réflexion sur ces questions, sans se laisser enfermer a priori dans les limites d'un modèle de développement incarné par le cadre de pensée de l'OMC, reprendre le problème à la racine, en partant des préoccupations des populations concernées; dynamiser les liens entre ONG de lobbying du Nord et organisations de terrain du Sud.

\section{Que faire face à la libéralisation des échanges?}

Quatre points, interdépendants, sont clairement ressortis des consultations avec la société civile des pays en développement.

1. Intégrer ou réintégrer le commerce dans des stratégies nationales de développement: ces stratégies nationales ne doivent pas être imposées de l'extérieur, mais décidées de manière endogène, en fonction des spécificités de chaque pays et des besoins de leurs populations. L'enjeu ici est le besoin et le droit des Etats du Sud comme du Nord de conserver, recréer ou se réapproprier un vrai "espace politique».

2. Repenser l'architecture du commerce international, à partir du plan local et régional: incontournables dans la mesure où aucun pays ne peut vivre en autosuffisance ni échapper à la mondialisation, les échanges commerciaux ne sont ni bons ni mauvais en soi. Les politiques économiques doivent viser en priorité le développement d'un marché national et l'intégration régionale, avant de chercher la participation à tout prix au marché mondial et la promotion des exportations.

3. Réformer I'OMC: les participants ont, dans leur grande majorité, souligné la nécessité d'une instance multilatérale de régulation du commerce international. Trois questions, liées à des propositions de réformes, ont notamment été discutées lors du symposium: meilleure utilisation des marges de manœuvre et flexibilités qui existent à I'OMC, création de structures et procédures plus transparentes et démocratiques, différentiation structurelle entre les pays intégrant mieux les niveaux de développement. Cette différenciation nécessaire ne doit cependant pas être confondue avec l'introduction d'une nouvelle catégorisation des pays en développement à l'OMC.

4. Elaborer et promouvoir des modèles d'économie et de commerce alternatifs: ces modèles doivent être capables de faire pièce au système dominant. Trois pistes ont notamment été évoquées. Une "alternative" ne signifie pas un retrait hors des marchés internationaux, mais un réenchâssement de l'économie dans la société. L'« essence» du développement consiste dans l'accroissement de la diversité des options et le respect pour les individus, les communautés, les sociétés et les nations de la liberté de choisir ce qu'ils estiment être le meilleur pour eux. II convient d'articuler d'une manière cohérente le commerce équitable "local et régional».

Voir Michel Egger, Se réapproprier un espace politique, Dossiers Global+ (Alliance Sud), n 5, 2005. Ce dossier présente une synthèse des résultats du symposium, complétée par les textes des différentes interventions en langue originale.

Les contributions ${ }^{52}$ des $\mathrm{ONG}$ du Sud reflètent non seulement une multiplicité d'expériences, mais aussi une diversité de points de vue. Dans leur état des lieux, toutes les organisations consultées sont cependant unanimes sur un point: le «credo néolibéral» - selon lequel la libéralisation des échanges et l'intégration au marché mondial constituent les clés du développement et de la lutte contre la pauvreté - serait une «mystification». Il ne correspond en rien à la réalité vécue des populations. Selon ces ONG, dix ans d'OMC et de pro-

52 Michel Egger, Quel commerce pour quel développement?, Dossiers Global+ (Alliance Sud), $\mathrm{n}^{\mathrm{o}} 4$, 2005. Ce dossier présente une compilation des 57 contributions des partenaires du Sud. 
grammes du Fonds monétaire international (FMI) ont, au contraire, surtout contribué à éroder la marge de manœuvre des Etats, augmenté l'emprise des multinationales, conduit à la privatisation des services publics, exacerbé les inégalités et détruit l'environnement.

Le problème, de facto, n'est pas le commerce international en soi, mais la manière dont les relations et les règles commerciales s'établissent. Celles-ci reflètent et renforcent les «asymétries existantes». On parle de «libre-échange» pour tous, mais seule une minorité en profite: les pays du Nord, les multinationales et les élites des pays en développement. Non seulement on met en compétition des acteurs totalement inégaux, mais on permet aux plus forts de protéger leurs secteurs sensibles et de perpétuer des pratiques déloyales.

\subsubsection{Colloque sur les services}

La Déclaration de Berne a organisé, le 6 décembre 2005 à Berne, un colloque international sur le thème «Libre accès au marché pour les banques: qui paie le prix ?» ${ }^{53}$. L'objectif était d'éclairer un chapitre encore peu exploré des négociations à l'OMC: la libéralisation des marchés financiers. Ce thème a, dès le départ, été très controversé à l'OMC. De nombreux pays en développement, notamment les pays émergents, se sont longtemps opposés à un accord, soulignant les inégalités structurelles entre pays du Nord et du Sud. Un tel accord a cependant été adopté fin 1997 - l'année de la crise financière asiatique - et est entré en vigueur en 1999.

Le secteur financier et bancaire figure parmi les intérêts prioritaires de la Suisse; sans en donner le détail, elle a fait des demandes importantes d'ouverture aux pays du Sud, tout comme l'Union européenne et les Etats-Unis. Ces pays sont convaincus qu'un marché financier libéralisé va stimuler la croissance économique et élever le niveau de vie de tous les membres de l'OMC, constituant par là même un élément clé du cycle de Doha. Le symposium a analysé et mis en discussion cette thèse ainsi que ses principaux arguments: innovation, transfert de technologies, baisse des coûts des prestations financières, amélioration de la compétitivité des sociétés locales. Il s'est notamment penché sur la politique des grandes banques étrangères dans les pays en développement, sur ses retombées pour l'économie bancaire locale et le crédit aux petites entreprises et aux populations dans les zones rurales, et sur sa contribution au cycle du développement de Doha.

53 Déclaration de Berne, page du colloque «Libre accès au marché pour les banques: qui paie le prix? », programme et textes des principales interventions : <www.evb.ch/fr/p10318.html >. 


\section{SOURCES}

Organisation mondiale du commerce (OMC)/World Trade Organization (WTO)

Conseil consultatif de l'OMC, L'avenir de l'OMC. Relever les défis institutionnels du nouveau millénaire, rapport du Conseil consultatif à l'ancien directeur général, Supachai Panitchpakdi, Genève, 2004.

Déclaration de S.E.M Joseph Deiss, conseiller fédéral, chef du Département fédéral de l'économie, doc. WT/MIN(05)/ST/2, 15 décembre 2005.

Déclaration ministérielle adoptée le 18 décembre 2005, doc. WT/MIN(05)/DEC, Genève, 2005.

Les pays les plus pauvres auront plus de temps pour appliquer les règles de propriété intellectuelle, communiqué de presse, no 424, 29 novembre 2005.

Protocole d'accession de la République populaire de Chine à l'OMC, 10 novembre 2001.

Rapport sur le commerce mondial 2005, Genève, 2005.

Statistiques du commerce international 2005, Genève, 2005.

Statistiques du commerce. La croissance du commerce devrait ralentir en 2005 par rapport au rythme record de 2004, communiqué de presse, nº 417, 27 octobre 2005.

Organisations non gouvernementales et groupes de pression

Alliance Sud/Déclaration de Berne/Pro Natura, Le paquet du développement est un leurre, communiqué de presse, 16 décembre 2005.

Alliance Sud/Déclaration de Berne/Pro Natura, Les ONG suisses déçues et inquiètes, communiqué de presse, 18 décembre 2005.

Coordination Suisse-OMC, La Coordination Suisse-OMC exige un vrai cycle du développement, communiqué de presse, 5 décembre 2005.

Déclaration de Berne, colloque «Libre accès au marché pour les banques: qui paie le prix ?», décembre 2005, programme et principales interventions, <www.evb.ch/fr/p10318.html >.

Egger M., Quel commerce pour quel développement?, Dossiers Global+ (Alliance Sud), n 4, 2005.

Egger M., Se réapproprier un espace politique, Dossiers Global+ (Alliance Sud), nº 5, 2005.

Economiesuisse, L'économie suisse favorable à une stratégie offensive pour la conférence ministérielle de l'OMC, communiqué de presse, 30 novembre 2005.

Economiesuisse, L'OMC n'a fait que des progrès minimaux à Hongkong, communiqué de presse, 19 décembre 2005

\section{SITES INTERNET}

ACICI (Agence de coopération et d'information pour le commerce international) : <www.acici.org $>$.

Alliance Sud: <www.alliancesud.ch $>$.

Banque mondiale: <www.worldbank.org $>$.

Campagne GATS et démocratie: $<w w w . a l l i a n c e s u d . c h>$ et $<w w w . s u i s s e$.attac.org $>$.

CNUCED : <www.unctad.org $>$.

Déclaration de Berne: <www.evb.ch $>$.

Fédération des entreprises suisses (economiesuisse) : <www.economiesuisse.ch $>$.

ICTSD (Centre international pour le commerce et le développement durable) : <www.ictsd.org $>$.

IDEAS Centre : <www.ideascentre.ch>.

Office of the United States Trade Representative : <www.ustr.gov>.

OMC: $<$ www.wto.org $>$.

OMC, dixième anniversaire : <www.wto.org/french/thewto_f/10anniv_f/10anniv_f.htm>.

ONU : <www.un.org >

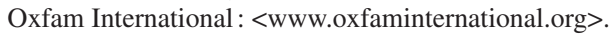

seco (Secrétariat à l'économie) : <www.seco-admin.ch $>$.

Semaine mondiale d'action sur le commerce: $<w w w$.april2005.org $>$.

Third World Network: <www.twnside.org.sg $>$.

Union africaine: $<w w w . u n e c a . o r g>$.

Union européenne: $<w w w . e u r o p a . e u . i n t>$.

Union suisse des paysans: <www.bauernverband.ch $>$. 